\title{
MASS TRANSFER CHARACTERIZATION IN CLOSE BINARY STARS
}

\author{
J. M. GARCÍA
}

Departamento de Física, Universidad Politécnica de Madrid

Ronda de Valencia, 3. 28012 Madrid, Spain.

\section{A. GIMÉNEZ}

Laboratorio de Astrofísica Espacial y Física Fundamental (INTA). Apartado 50727. 28080 Madrid, Spain.

\begin{abstract}
A method for estimating representative values of the parameters that characterize the loss of mass and angular momentum occurred during the evolution of a close binary star is considered. Absolute dimensions previous to mass-transfer stage have been obtained for a select group of 43 semidetached systems adopting a grid of values of mass loss, angular momentum loss and initial mass ratio. Our study reveals a highly non-conservative scenario for the evolution towards an Algol-type system.
\end{abstract}

\section{Introduction}

The accurate knowledge of the evolution that takes place in a close binary system, when the more massive component reaches its Roche's lobe, requires the establishment of the form in which mass and angular momentum changes.

Different studies up to now have shown that the conservative approach (total mass and total angular momentum remain constant) is not compatible with the observed characteristics of a good number of Algol type binaries.

Using the new data for 43 semidetached systems, that we present in other communication to this Symposium (García and Giménez, 1991), we have carried out an analysis of the most probable values of the parameters $\alpha$ and $f$, which characterize the loss of angular momentum and mass in a detached close binary star, in its evolution towards an Algol-type configuration.

\section{Detached and semidetached binaries in the period-mass diagram}

We have compared the distribution of a group of detached binaries in the diagram $\log \mathrm{P}-\log \mathrm{m}$ with the progenitors of the 43 semidetached systems mentioned above.

To calculate the masses and the periods of the progenitor stars we have adopted the non conservative approach of Vanveberen et al. (1979) and the grid of values $\alpha \in[0.0,4.0]$ and $f \in[0.0,1.0]$. The calculations have been repeated for different values of the initial mass ratio, $q_{0} \in[1.05,2.0]$.

The chosen sample of detached binaries consists of 46 stars with well-known dimensions taken from Popper (1980), Harmanec (1988) and from other studies on individual systems published more recently. The evolution of a detached binary according to case A or case B, depends essentially on the mass of its components and on its period. The period which stablish the limit between the $A$ and $B$ cases of mass transfer depends on the initial $q_{0}$ according to the expression:

$$
\log P_{A B}=1.5 \log \frac{R_{1 T}}{r_{1 L}^{*}}-0.5 \log \left(m_{1}+\frac{m_{1}}{q_{O}}\right)-0.936
$$


where $r_{1 \mathrm{~L}}^{*}$ is the equivalent radius of the Roche lobe of the primary component, $R_{1 \mathrm{~T}}$ is the absolute radius that this star, of mass $m_{1}$, has when it reaches the TAMS and $q_{0}=m_{1} / m_{2}$.

For the calculation of $\dot{r}_{1 \mathrm{~L}}^{*}$ we have taken the equation given by Eggleton (1983), while $R_{1 \mathrm{~T}}$ was taken from the standard evolutionary models of Claret and Gimenez (1989).

From figure 1 it follows that $96 \%$ of the detached systems will evolve according to case B and the remaining $4 \%$ according to case $\mathrm{A}$. The same percentages should be found among the progenitor stars of those which are observed as Algol-type systems at present. In other words, the distributions upon the period-mass diagram must be similar.

For each adopted group of values $\left(\alpha, \mathrm{f}, \mathrm{q}_{0}\right)$, we have evaluated the existing differences between both distributions according to several factors: $a$ ) difference in the percentage of $B$ cases; $b$ ) difference in the percentage of A cases; $c$ ) mass distribution; d) presence of progenitors in the forbidden region (below the ZAMS line).

Figure 2 represents the above mentioned differences for three selected values of $q_{0}$ and the complete grid of $\alpha$ and $\mathrm{f}$. As it was expected, in the three cases the distribution of detached systems becomes very different from that of the progenitors when physically unrealistic situations are considered. That is the case of a high loss of mass together with a minimal loss of angular momentum.

We have verified that a trend exists for the systems with small observed $q$ (often classified as $\mathrm{R} \mathrm{CMa)} \mathrm{to} \mathrm{be} \mathrm{placed} \mathrm{in} \mathrm{the} \mathrm{forbidden} \mathrm{region.} \mathrm{This} \mathrm{may} \mathrm{show} \mathrm{that} \mathrm{such} \mathrm{systems} \mathrm{have} \mathrm{evolved} \mathrm{under}$ extreme conditions of mass loss or that $\mathrm{q}_{0}$ is much greater than for the rest of the systems. Nevertheless we have repeated the calculations by removing from the list of semidetached binaries the $\mathrm{R} \mathrm{CMa}$ type systems. We found out that the most appropriate values for $f$ and $\alpha$, are the same as in the previous situation.

From figure 2 it can be concluded that the mass loss is in any case higher than $30 \%$ (f $>0.3$ ) and the associated loss of angular momentum is rather high, being characterized by $\alpha>2.5$. It is noticed a trend to the decrease of the optimal values of $f$ and $\alpha$ when the assumed initial mass ratio increases. Nevertheless, most of the considered detached binaries have $q \leq 1.25$, so that

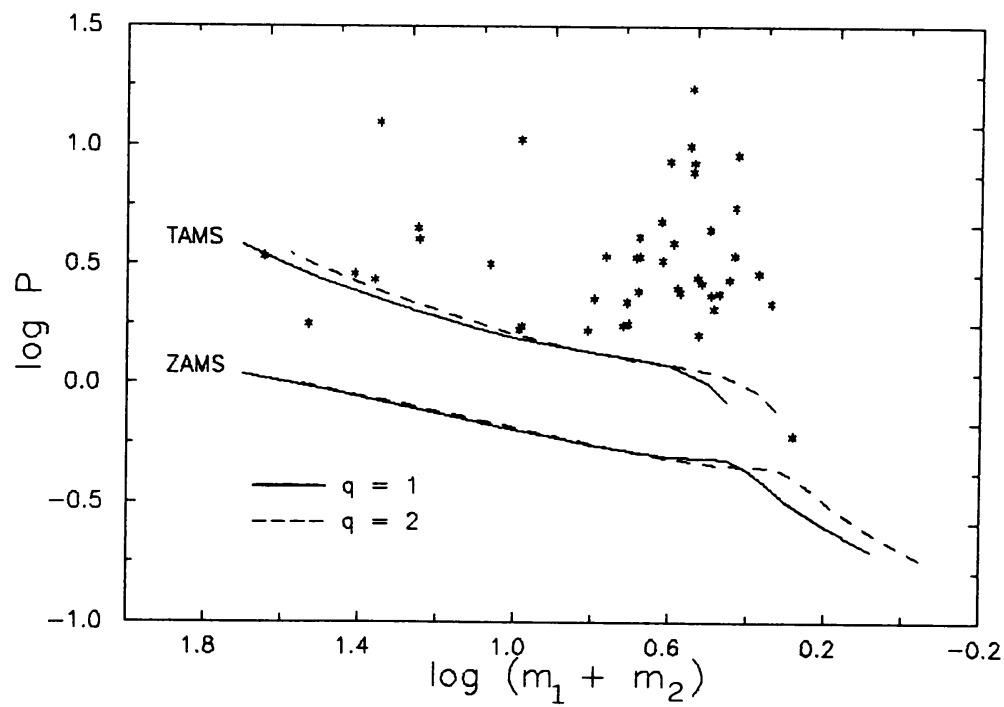

Figure 1. Logarithm of the orbital period versus the logarithm of the total mass for the 46 detached systems considered. The line denoted with TAMS is the limit between the A and B cases of mass transfer. 


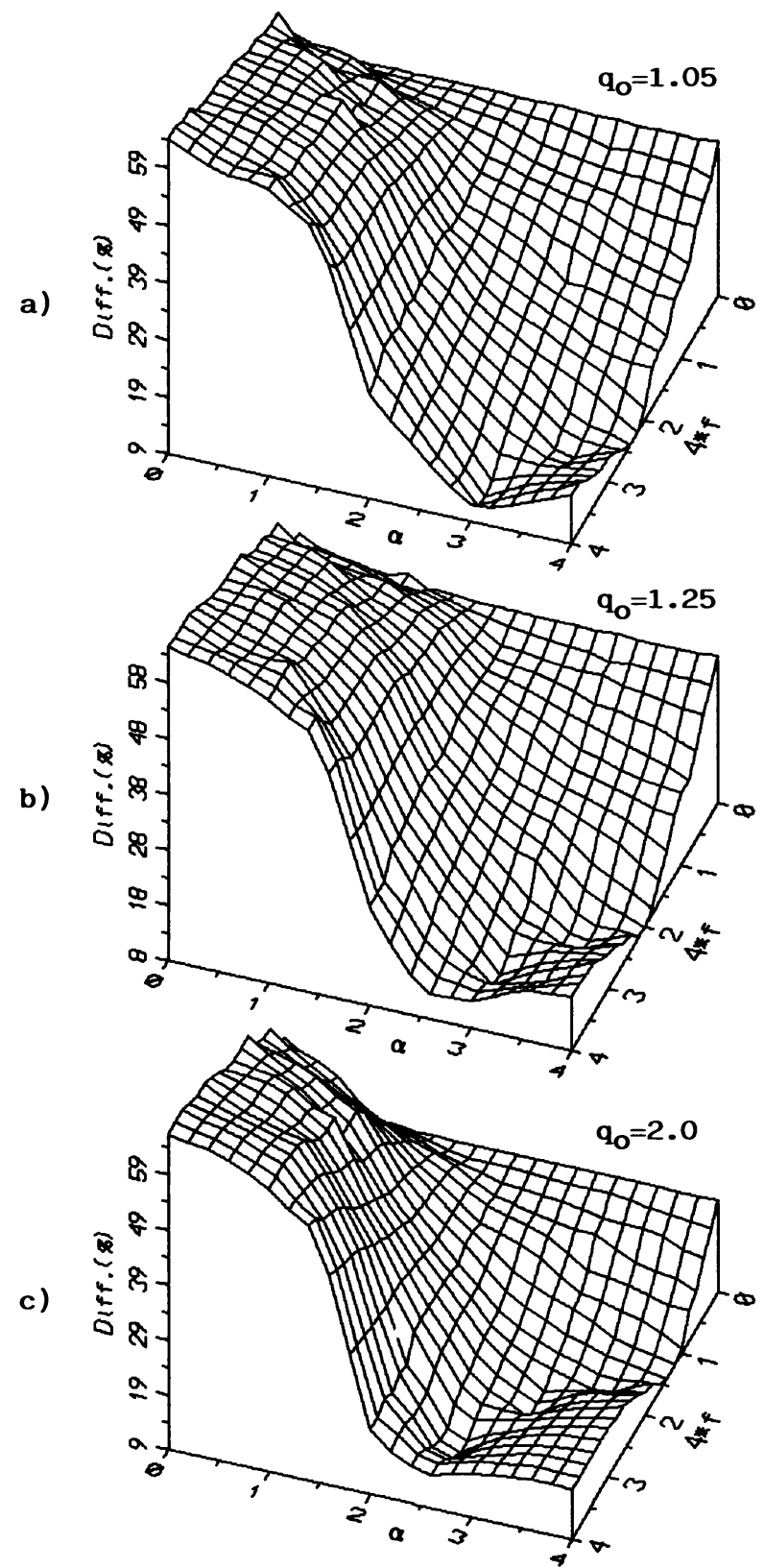

Figure 2. Differences between the distribution of detached binaries upon the mass-period diagram and that of the progenitors of semidetached systems. Each plot corresponds to a selected value of the assumed initial mass ratio, $q_{0}$, and to the complete grid of values of $f$ and $\alpha$. 
if we suppose $q_{0}$ significantly higher than this value to calculate the masses and periods of the progenitor: systems, both samples are no longer comparable. Consequently only the results derived from figure 2 corresponding to $\mathrm{q}_{0}=1.05$ and $\mathrm{q}_{0}=1.25$, have been considered.

According to the minima which appear on these diagrams, we can estimate the most probable values of mass and angular momentum lost within the ranges $\alpha \sim 3.5-4.0$ and $f \sim 0.6-0.7$.

Both distributions become very different when the conservative case $(\alpha=0, f=0)$ or close situations are considered. Figure 3 shows the progenitors of the present semidetached systems whose dimensions were obtained adopting $\alpha=3.5$ and $\mathrm{f}=0.7$. The distribution for mass and period is in that case very similar to that of figure 1 , which corresponds to the group of presently detached stars.

We are well aware of the fact that each system had its own different $q_{0}$ and of the presence of observational selection effects, therefore the global analysis assuming the same initial mass ratio for all of them, has to be handled with care. The results obtained correspond to a mean value of the $\alpha$ and $\mathrm{f}$ parameters.

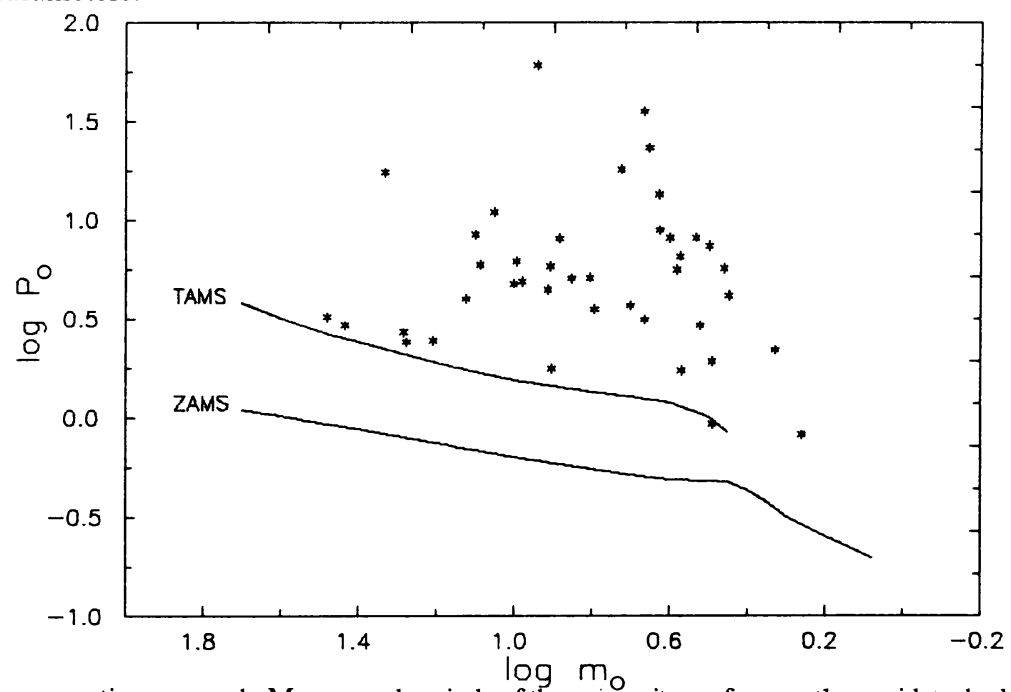

Figure 3. Non-conservative approach. Masses and periods of the progenitors of presently semidetached binaries are calculated by assuming $\mathrm{f}=0.7, \alpha=3.5$ and $\mathrm{q}_{0}=1.1$

\section{Acknowledgements}

This work has been supported by the Spanish DGICYT through project PB87-0235

\section{References}

Claret,A. and Giménez. A. (1989), Astron. Astrophys. Suppl. 81, 1.

Eggleton, P.P. ( 1983), Astrophys. J. 268, 368.

García, J.M. and Giménez, A. ( 1991), "A New Catalogue of Semidetached Algol-type Binaries with Well-Determined Absolute Parameters", Communication to this Symposium.

Harmanec, P. ( 1988), Bull. Astron. Inst. Czech. 39, 329.

Popper, D.M. ( 1980), Ann. Rev. Astron. Astrophys. 18, 115.

Vanveberen, D., De Greve, J.P., Van Dessel, E.L., De Loore,C. (1979), Astron. Atrophys. 73, 19. 